\title{
First report of a crinivirus infecting mulberry (Morus alba L.) in China
}

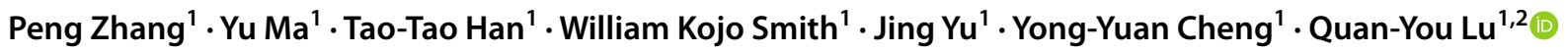

Received: 14 August 2021 / Accepted: 15 November 2021 / Published online: 29 November 2021

(c) Società Italiana di Patologia Vegetale (S.I.Pa.V.) 2021

Keywords Morus alba $\mathrm{L} \cdot$ Mulberry crinivirus $\cdot$ Crinivirus

Mulberry viral disease is one of the most important limiting factors of the sericultural industry. In 2019, a virus-focused study was performed in a mulberry field in Zhenjiang, Jiangsu Province, China. Leaves of one of mulberry plants showing virus-like symptoms with mosaic, mottling and dwarfism were collected for high-throughput sequencing (HTS) of small RNAs. The HTS results showed that a total of 128 contigs were related to known plant viral sequences. Seventy-one of these contigs were the genomic sequence of mulberry mosaic leaf roll-associated virus (Lu et al. 2015). The other 57 were associated with the possible new member of the genus Crinivirus in the family Closteroviridae tentatively named mulberry crinivirus (MuCV). To fill the gaps between the contigs, dsRNA was extracted from leaves of the original plant and used as a template for reverse transcription (RT) and polymerase chain reactions (PCRs) with the specific primers designed on the contigs sequences. Amplicons were ligated into $\mathrm{pUCm}-\mathrm{T}$ vector and at least 3 clones per sample were sequenced. A $6799 \mathrm{nt}$ sequence of RNA2 was finally determined (GenBank accession No. MW741881). This partial sequence contains the complete ORFs coding for $\mathrm{p} 59$, major coat protein $(\mathrm{CP})$ and minor $\mathrm{CP}(\mathrm{CPm})$, as well as partial cistrons encoding heat shock protein 70 homolog (Hsp70h) and p26 proteins. A BLASTx search indicated that amino acid sequences of $\mathrm{Hsp} 70 \mathrm{~h}$ and CP of MuCV shared the highest identify $(68.75 \%, 54.69 \%$ respectively) with those of jasmine virus A-1 (MN915110). According to the species demarcation criteria for the genus Crinivirus

Quan-You Lu

sriluqy@163.com

1 College of Biotechnology, Jiangsu Key Laboratory of Sericultural Biology and Biotechnology, Jiangsu University of Science and Technology, Zhenjiang 212018 , Jiangsu, China

2 Key Laboratory of Genetic Improvement of Silkworm and Mulberry, Ministry of Agriculture and Rural Affairs, Sericultural Research Institute, Chinese Academy of Agricultural Sciences, Zhenjiang 212018, Jiangsu, China proposed by International Committee on Taxonomy of Viruses (Fuchs et al. 2020), MuCV is a new member of the genus Crinivirus. To assess the occurrence of MuCV, RT-PCR was performed using MuCV-specific primers (forward, 5'-CAACAA TGGTGCCGGTGCGAAT-3', and reverse, 5'-TTAGTTCTA CAACCATTGGTG-3') designed on CP gene sequences. Ten out of forty-three randomly selected mulberry samples from the same filed were MuCV positive. To the best of our knowledge, this is the first report of mulberry as a host of crinivirus.

Supplementary information The online version contains supplementary material available at https://doi.org/10.1007/s42161-021-01017-7.

Funding This work was partially granted by the Natural Science Foundation of the Jiangsu Higher Education Institutions of China (No. 18KJA210001)

Data availability The data (DNA sequences) presented in the manuscript have been deposited in GenBank database under accession number MW741881.

\section{Declarations}

This article does not contain any studies with human participants or animals performed by any of the authors.

Conflict of interest The author(s) declare no conflict of interest.

\section{References}

Fuchs M, Bar-Joseph M, Candresse T, Maree HJ, Martelli GP, Melzer MJ, Menzel W, Minafra A, Sabanadzovic S (2020) ICTV virus taxonomy profile: Closteroviridae. J Gen Virol 101:364-365

Lu QY, Wu ZJ, Xia ZS, Xie LH (2015) A new nepovirus identified in mulberry (Morus alba L.) in China. Arch Virol 160:851-855

Publisher's Note Springer Nature remains neutral with regard to jurisdictional claims in published maps and institutional affiliations. 